\title{
Breast Myoid Hamartoma
}

National Cancer Institute

\section{Source}

National Cancer Institute. Breast Myoid Hamartoma. NCI Thesaurus. Code C40404.

A breast hamartoma characterized by the predominance of a smooth muscle component. 